nternational Journal of Bifurcation and Chaos, Vol. 17, No. 12 (2007) 4481-4492

(C) World Scientific Publishing Company

\title{
AUTHOR INDEX VOLUME 17 (2007)
}

Abel, M. \& Bergweiler, S., Synchronization of higher harmonics in coupled organ pipes

Acho, L., see Benitez

Adamatzky, A., Phenomenology of retained excitation

Adomaitis, R.A., Kevrekidis, I.G. \& de la Llave, R., A computer-assisted study of global dynamic transitions for a noninvertible system

Adomaitis, R.A., The trouble with spurious eigenvalues

Aihara, K., see Takemoto

Aihara, K., see Tsuji

Aihara, K., see Wang

Akatsu, S., Torikai, H. \& Saito, T., Zero-cross instantaneous state setting for control of a bifurcating H-bridge inverter

Albert, R., see Christensen

Alberto, L.F.C. \& Chiang, H.-D., Uniform approach for stability analysis of fast subsystem of two-time scale nonlinear systems

Algaba, A., Freire, E., Gamero, E. \& RodríguezLuis, A.J., Resonances of periodic orbits in Rössler system in presence of a triple-zero bifurcation

Ali, M., see Litak

Allefeld, C., Müller, M. \& Kurths, J., Eigenvalue decomposition as a generalized synchronization cluster analysis

Amemiya, T., see Yamamoto

Amemiya, Y., see Kikombo

Amemiya, Y., see Takahashi

Anosov, O., Hensel, B., Berczynski, S. \& Kravtsov, Yu.A., Detection threshold of the control parameters modulation in a noisy chaotic map

Antoneli, F. \& Stewart, I., Symmetry and synchrony in coupled cell networks 2: Group networks

Arecchi, F.T., see Mariño

Arena, P., Fortuna, L., Frasca, M., Lombardo, D., Patanè, L. \& Crucitti, P., Turing patterns in RD-CNNs for the emergence of perceptual states in roving robots

Arrowsmith, D.K., see Gutiérrez

Asai, T., see Kikombo

Asai, T., see Oya

Asai, T., see Takahashi

Astolfi, A., see Tigan

Atanov, N., see Dmitriev
Athias, P., see Jacquir $\quad 4417$

3483

617

3985

1305

1375

459

985

3085

3571

2201

4195

1997

2797

3493

3679

3613

1713

1661

935

3639

Bakri, T., On the modified logistic equation

2541

Balthazar, J.M., see Cassiano

Banks, S.P., see Song

Banks, S.P., see Song

Bánhelyi, B., Csendes, T. \& Garay, B.M., Optimization and the Miranda approach in detecting horseshoe-type chaos by computer

Baptista, M.S., see Pereira

Baranovski, A.L. \& Lawrance, A.J., Sensitive parameter dependence of autocorrelation function in piecewise linear maps

Barbaro, G., Formal solutions of the CvitanovicFeigenbaum equation

Barboza, R., Dynamics of a hyperchaotic Lorenz system

Barbé, A. \& von Haeseler, F., Correlation and spectral properties of multidimensional ThueMorse sequences

Barra, F., Stress driven interface dynamics: The effects of surface stress

Barrat, A., see Colizza

Barthélemy, M., see Colizza

Bauernschmitt, R., see Wessel

Beaver, T.M., see Simonotto

Belykh, I., Hasler, M. \& Belykh, V., When symmetrization guarantees synchronization in directed networks

Belykh, V., see Belykh

Benítez, S. \& Acho, L., Impulsive synchronization for a new chaotic oscillator

Berczynski, S., see Anosov

Bergweiler, S., see Abel

Bilbault, J.-M., see Jacquir

Bilbault, J.M., see Jacquir

Billings, S.A., see Guo

Billings, S.A., see Pan

Billings, S.A., see Zhao

Billings, S.A., see Zhao

Bilotta, E., di Blasi, G., Stranges, F. \& Pantano, P., A gallery of Chua attractors. Part IV

Bilotta, E., Di Blasi, G., Stranges, F. \& Pantano, P., A gallery of Chua attractors. Part VI

Bilotta, E., Di Blasi, G., Stranges, F. \& Pantano, P., A gallery of Chua attractors. Part $V$

Bilotta, E., Pantano, P. \& Stranges, F., A gallery of Chua attractors: Part I

Bilotta, E., Pantano, P. \& Stranges, F., A gallery of Chua attractors: Part II
2009

2073

2085

735

3545

1185

3275

4285

1721

2491

2491

3325

3229

3387

3387

617

1661

3483

3697

4417

1985

4323

153

1687

1017

1801

1383

1

293 
Bilotta, E., Stranges, F. \& Pantano, P., A gallery of Chua attractors: Part III

Binczak, S., see Jacquir

Binczak, S., see Jacquir

Blomgren, P., see Gasner

Blomgren, P., see Palacios

Boccaletti, S., Hwang, D.-U. \& Latora, V., Growing hierarchical scale-free networks by means of nonhierarchical processes

Boccaletti, S., see Brugioni

Bollt, E., Attractor modeling and empirical nonlinear model reduction of dissipative dynamical systems

Bondarenko, V.E. \& Rasmusson, R.L., Suppression of cellular alternans in guinea pig ventricular myocytes with LQT2: Insights from the Luo-Rudy model

Bonnin, M., Corinto, F. \& Gilli, M., Bifurcations, stability and synchronization in delayed oscillatory networks

Bonnin, M., see Corinto

Bossu, J., see Morfu

Boukabou, A. \& Mansouri, N., Fuzzy predictive controller for unknown discrete chaotic systems

Bountis, T., see Mahmoud

Bowong, S., see Moukam Kakmeni

Bowong, S., see Yamapi

Brandes, U. \& Pich, C., Centrality estimation in large networks

Braunstein, L.A., Wu, Z., Chen, Y., Buldyrev, S.V., Kalisky, T., Sreenivasan, S., Cohen, R., López, E., Havlin, S. \& Stanley, H.E., Optimal path and minimal spanning trees in random weighted networks

Broer, H.W., Ciocci, M.C. \& Hanßmann, H., The quasi-periodic reversible Hopf bifurcation

Brugioni, S., Hwang, D.U., Meucci, R. \& Boccaletti, S., Coherence resonance in a FitzhughNagumo electronic system

Buldyrev, S.V., see Braunstein

Buldú, J.M., García-Ojalvo, J., Wagemakers, A. \& Sanjuán, M.A.F., Electronic design of synthetic genetic networks

Buldú, J.M., see Park

Bumelienè, S., see Lindberg

Bumelienè, S., see Tamaševičius

Bungay, S.D. \& Campbell, S.A., Patterns of oscillation in a ring of identical cells with delayed coupling

Buscarino, A., Fortuna, L., Frasca, M. \& Muscato, G., Chaos does help motion control

Buscarino, A., Fortuna, L., Frasca, M. \& Rizzo, A., Effects of long-range connections in distributed control of collective motion

Cabeza, C. \& Rosen, M., Complexity in Faraday experiment with viscoelastic fluid

Cafagna, D. \& Grassi, G., Decomposition method for studying smooth Chua's equation with application to hyperchaotic multiscroll attractors

Caldarelli, G., see Ferrer $i$ Cancho

Caldas, I.L., see Portela

4067

657

3697

4417

2765

509

2447

3431

1199

381

4033

4365

3535

2141

4295

3259

1775

2303

2215

2605

3431

2215

3507

2281

3449

3657

3109

3577

2411

1599

209

2453
Caldas, I.L., see Portela

1589

Calude, C.S. \& Dinneen, M.J., Exact approximations of omega numbers

Campbell, S.A., see Bungay

Campos, C.D., Palhares, R.M., Mendes, E.M.A.M., Torres, L.A.B. \& Mozelli, L.A., Experimental results on Chua's circuit robust synchronization via LMIs

Campos-Cantón, E., González, J.S. \& Urías, J., Poincaré planes in nonlinear electronics

Cano, P., see Park

Cao, J., see Huang

Cao, J., see Huang

Cao, J., see Xiao

Cao, J., see $Y u$

Capocci, A., see Ferrer $i$ Cancho

Cassiano, J. \& Balthazar, J.M., On homoclinic chaos and a control chaos strategy applied to a free-joint nonholonomic manipulator

Castellani, G.C., see Remondini

Castro, V., Monti, M., Pardo, W.B., Walkenstein, J.A. \& Rosa, Jr., E., Characterization of the Rössler system in parameter space

Celma, O., see Park

Chacón, R., García-Hoz, A.M. \& Martínez, J.A., Reshaping-induced control of chaos and crisis phenomena in a damped, parametrically driven pendulum

Chaitin, G., How much information can there be in a real number?

Chan, W.-C.C. \& Lin, S.-S., Existence and stability of multibump solutions of an integraldifferential equation

Chang, P.J. \& Muthuswamy, B., Optimal CNN templates for linearly-separable onedimensional cellular automata

Chang, S.-L. \& Chien, C.-S., Numerical continuation for nonlinear Schrödinger equations

Chang, Y.-W., Juang, J. \& Li, C.-L., Snap-back repellers and chaotic traveling waves in onedimensional cellular neural networks

1937

3109

3199

199

2281

2723

4159

4309

1355

2453

2009

2477

965

2281

625

1933

4099

749

641

Chen, F., see Guan

Chen, F., see Lou

Chen, F.-J., Li, J.-B. \& Chen, F.-Y., Chaos for discrete-time RTD-based cellular neural networks

Chen, F.-Y., see Chen

Chen, G., see Chen

Chen, G., see Han

Chen, G., see Liu

Chen, G., see $L i$

Chen, G., see Luo

Chen, G., see Robbio

Chen, G., see Shi

Chen, G., see Yang

Chen, G., see $Y u$

Chen, G., see $Y u$

Chen, G., see $Y u$

Chen, G., see Zhang

Chen, G., see Zhang
1969

4245

4145

4395

4395

243

2033

4153

4049

3235

1623

4355

3929

1785

2791

3951

61

4443 
Chen, G., see Zhou

Chen, L., see Gao

Chen, Q., Zhong, Q., Hong, Y. \& Chen, G., Generation and control of spherical and circular attractors using switching schemes

Chen, T. \& Zhu, Z., Exponential synchronization of nonlinear coupled dynamical networks

Chen, T., see Zhang

Chen, Y., Tse, C.K., Wong, S.-C. \& Qiu, S.-S., Interaction of fast-scale and slow-scale bifurcations in current-mode controlled $D C / D C$ converters

Chen, Y., see Braunstein

Chengdong, D., see Zengrong

Chernihovskyi, A. \& Lehnertz, K., Measuring synchronization with nonlinear excitable media

Chiang, H.-D., see Alberto

Chien, C.-S., see Chang

Chlouverakis, K.E., see Sprott

Chou, M.-H., Wei, H.-C., Lin, Y.-T., Oregonatorbased simulation of the Belousov-Zhabotinskii reaction

Christensen, C. \& Albert, R., Using graph concepts to understand the organization of complex systems

Chua, L.O., Guan, J., Sbitnev, V.I. \& Shin, J., A nonlinear dynamics perspective of Wolfram's new kind of science. Part VII: Isles of Eden

Chua, L.O., Karacs, K., Sbitnev, V.I., Guan, J. \& Shin, J., A nonlinear dynamics perspective of Wolfram's new kind of science. Part VIII: More isles of Eden

Chua, L.O., see Itoh

Chua, L.O., see Itoh

Chua, L.O., see Itoh

Ciocci, M.C., see Broer

Cisternas, J. \& Descalzi, O., Sources and sinks in the vicinity of a weakly inverted instability

Cohen, R., see Braunstein

Colacchio, G., Sparro, M. \& Tebaldi, C., Sequences of cycles and transitions to chaos in a modified Goodwin's growth cycle model

Colizza, V., Barrat, A., Barthélemy, M. \& Vespignani, A., Epidemic predictability in metapopulation models with heterogeneous couplings: The impact of disease parameter values

Constantinescu, F., Nitescu, M. \& Ionescu, A., When is shooting appropriate in steady state analysis of nonlinear circuits

Corbera, M. \& Llibre, J., Generation of symmetric periodic orbits by a heteroclinic loop formed by two singular points and their invariant manifolds of dimensions 1 and 2 in $R^{3}$

Corinto, F., Bonnin, M. \& Gilli, M., Weakly connected oscillatory network models for associative and dynamic memories

Corinto, F., see Bonnin

Coronnello, C., see Tumminello

Costa, da F.L. \& Sporns, O., Diversity of cortical states at nonequilibrium simulated by the

999

1609

2215

2109

3425

4195

641

2097

2839

anti-ferromagnetic Ising model under metropolis dynamics

Criado, R., Hernández-Bermejo, B. \& Romance, M., Efficiency, vulnerability and cost: An overview with applications to subway networks worldwide

Crucitti, P., see Arena

2289

107

735

4409

Cui, B., see Lou

D'Amico, M.B., see Zhang

Dana, S.K. \& Roy, P.K., Bursting near homoclinic bifurcation in two coupled Chua oscillators

61

Danca, M.-F., see Luo

Davis, M.S., Nutter, N.G. \& Rosa, Jr., E., Driving phase synchronous plasma discharges with superimposed signals

Dębiec, P., see Ślot

de la Llave, R., see Adomaitis

De Los Rios, P. \& Petermann, T., Existence, cost and robustness of spatial small-world networks

4337 Deneubourg, J.-L., see Ramírez Ávila

Deng, W., Generating 3-D scroll grid attractors of fractional differential systems via stair function

Dercole, F., Loiacono, D. \& Rinaldi, S., Synchronization in ecological networks: A byproduct of Darwinian evolution?

Descalzi, O., see Cisternas

di Bernardo, M., Garofalo, F. \& Sorrentino, F., Effects of degree correlation on the synchronization of networks of oscillators

di Bernardo, M., see Sorrentino

3741

427

1109

1531

2605

2821

2215

1911

2491

3583 di Blasi, G., see Bilotta

Di Blasi, G., see Bilotta

Di Blasi, G., see Bilotta

Di Marco, M., Forti, M., Grazzini, M., Nistri, P. \& Pancioni, L., Global consistency of decisions and convergence of competitive cellular neural networks

di Sciascio, E., see Grassi

Di Sciascio, E., see Grassi

Diaz, D., see Song

Dichmann, D.J., see Doedel

Dinneen, M.J., see Calude

Ditto, W.L., see Jahed-Motlagh

Ditto, W.L., see Simonotto

Dmitriev, A., Efremova, E., Kuzmin, L. \& Atanov, N., Forming pulses in nonautonomous chaotic oscillator

Doedel, E.J., Romanov, V.A., Paffenroth, R.C., Keller, H.B., Dichmann, D.J., Galán-Vioque, J. \& Vanderbauwhede, A., Elemental periodic orbits associated with the libration points in the circular restricted 3-body problem

Dolzmann, K., Kaltenbach, S., Hanke, W. \& Fernandes de Lima, V.M., Biphasic optical signal of an oscillating nonstationary BelousovZhabotinsky bulk reaction and its similarity to some neurophysiological events

Donner, R. \& Witt, A., Temporary dimensions of multivariate data from paleoclimate
3437

3235

3513

3171

1305

2331

4453

3965

2435

2821

3499

2419

1017

1383

1801

3127

1323

1703

2073

2625

1937

1955

3229 
records - novel measure for dynamic characterization of long-term climate change

Donner, R., Feudel, F., Seehafer, N. \& Sanjuán, M.A.F., Hierarchical modeling of a forced Roberts dynamo

Duan, Z., Wang, J., Li, R. \& Huang, L., A generalization of smooth Chua's equations under Lagrange stability

Dullin, H.R., Schmidt, S., Richter, P.H. \& Grossmann, S.K., Extended phase diagram of the Lorenz model

Dumitriu, L. \& Iordache, M., Numerical steadystate analysis of nonlinear analog circuits driven by multitone signals

Dumitriu, L., see Iordache

Dzhanoev, A. \& Loskutov, A., Stabilization of chaotic behavior in the restricted three-body problem

Efremova, E., see Dmitriev

Elwakil, A.S., see Radwan

England, J.P., Krauskopf, B. \& Osinga, H.M., Computing two-dimensional global invariant manifolds in slow-fast systems

Enjieu Kadji, H.G., see Yamapi

Fan, D., see Wei

Fan, H., see Wang

Fay, D. \& Ringwood, J., A wavelet transfer model for time series forecasting

Fernandes de Lima, V.M., see Dolzmann

Ferragut, A., Llibre, J. \& Teixeira, M.A., Hyperbolic periodic orbits from the bifurcation of a four-dimensional nonlinear center

Ferrer i Cancho, R., Capocci, A. \& Caldarelli, G., Spectral methods cluster words of the same class in a syntactic dependency network

Feudel, F., see Donner

Flammini, A., see Fortunato

Forti, M., see Di Marco

Fortuna, L., Frasca, M., Umana, E., La Rosa, M., Nicolosi, D. \& Sicurella, G., Organic Chua's circuit

Fortuna, L., see Arena

Fortuna, L., see Buscarino

Fortuna, L., see Buscarino

Fortunato, S. \& Flammini, A., Random walks on directed networks: The case of pagerank

Franceschi, C., see Remondini

Frasca, M., see Arena

Frasca, M., see Buscarino

Frasca, M., see Buscarino

Frasca, M., see Fortuna

Freire, E., Ponce, E. \& Ros, J., A biparametric bifurcation in $3 D$ continuous piecewise linear systems with two zones. Application to Chua's circuit

Freire, E., see Algaba

Freire, E., see Muñoz-Almaraz

Fujii, H., see Tsuji

Furman, M.D., see Simonotto

Galán-Vioque, J., see Doedel

Galán-Vioque, J., see Muñoz-Almaraz
Galias, Z. \& Zgliczyński, P., Infinite dimensional Krawczyk operator for finding periodic orbits of discrete dynamical systems

Gallas, J.A.C., see Lind

Gamero, E., see Algaba

Gao, S., Chen, L., Teng, Z. \& Xie, D., Dynamic complexities in an epidemic model with birth pulses and pulse culling

Garay, B.M., see Bánhelyi

García-Ojalvo, J., see Buldú

García-Hoz, A.M., see Chacón

Garofalo, F., see di Bernardo

Garofalo, F., see Sorrentino

Gasner, S., Blomgren, P. \& Palacios, A., Noise-induced intermittency in cellular patternforming systems

Gasner, S., see Palacios

Ge, W., see Gui

Giannerini, S., Rosa, R. \& Gonzalez, D.L., Testing chaotic dynamics in systems with two positive Lyapunov exponents: A bootstrap solution

4261

3461

1997

521

735

3507

625

3499

2419

2765

509

2127

Gidea, M. \& Masdemont, J.J., Geometry of homoclinic connections in a planar circular restricted three-body problem

Gilli, M., see Bonnin

Gilli, M., see Corinto

Giné, J., On the centers of planar analytic differential systems

Goh, K.-I., see Lee

Golubitsky, M. \& Krupa, M., Stability computations for nilpotent Hopf bifurcations in coupled cell systems

Gómez-Gardeñes, J. \& Moreno, Y., Synchronization of networks with variable local properties

1151

4033

4365

3061

2485

2595

2501

Gontar, V. \& Grechko, O., Special properties of images and corresponding signals generated by chemical reactions discrete chaotic dynamics

Gonzalez, D.L., see Giannerini

González, J.S., see Campos-Cantón

González-Miranda, J.M., Complex bifurcation structures in the Hindmarsh-Rose neuron model

Gotoda, H. \& Saso, Y., Unsteady motion of pool fire on small-scale burner

Grassi, G. \& Miller, D.A., Projective synchronization via a linear observer: Application to time-delay, continuous-time and discrete-time systems

Grassi, G., Vecchio, P., di Sciascio, E. \& Grieco, L.A., Cellular neural networks for edge detection

Grassi, G., Vecchio, P., Grieco, L.A. \& Di Sciascio, E., Cellular neural networks for video compression: An object-oriented approach

3415

169

199

3071

2185

1337

Grassi, G., see Cafagna

Grazzini, M., see Di Marco

Grechko, O., see Gontar

Grete, P. \& Markus, M., Residence time distributions for double-scroll attractors

Grieco, L.A., see Grassi
1703 
Grieco, L.A., see Grassi

Grossmann, S.K., see Dullin

Grosu, I., General coupling for mutual synchronization of three identical oscillators

Grygiel, K., see Śliwa

$\mathrm{Gu}$, E.-G., The feasible domains and their bifurcations in an extended logistic model with an external interference

Guan, J., Shen, S., Tang, C. \& Chen, F., Extending Chua's global equivalence theorem on Wolfram's new kind of science

Guan, J., see Chua

Guan, J., see Chua

Guan, J., see Lou

Gui, Z. \& Ge, W., Impulsive effect of continuoustime neural networks under pure structural variations

Guisset, J.-L., see Ramírez Ávila

Günel, S., see Hillier

Guo, L.Z. \& Billings, S.A., Identification of binary cellular automata from spatio-temporal binary patterns using a Fourier representation

Guo, Q. \& Li, C., Hopf bifurcation of a delayed differential equation

Gutiérrez, E. \& Arrowsmith, D.K., The equivalence between feedback and dissipation in impact oscillators

Haase, M., see Lind

Han, M., Chen, G. \& Sun, C., On the number of limit cycles in near-Hamiltonian polynomial systems

Han, M., Shang, D., Zheng, W. \& Yu, P., Bifurcation of limit cycles in a fourth-order nearHamiltonian system

Han, M., see Jiang

Han, X., see Wen

Hane, R. \& Kohda, T., Cryptanalysis of chaosbased ElGamal public-key encryption

Hanßmann, H., see Broer

Hanke, W., see Dolzmann

Harlim, J. \& Langford, W.F., The Cusp-Hopf bifurcation

Hasler, M., see Belykh

Havlin, S., see Braunstein

Heidel, J. \& Zhang, F., Nonchaotic and chaotic behavior in three-dimensional quadratic systems: Five-one conservative cases

Hensel, B., see Anosov

Henttu, P., see Montalbán

Hernández-Bermejo, B., see Criado

Hillier, D., Günel, S., Suykens, J.A.K. \& Vandewalle, J., Partial synchronization in oscillator arrays with asymmetric coupling

Hirata, Y., see Nakamura

Hirose, T., see Takahashi

Hogan, S.J., see Homer

Holzhüter, T. \& Klinker, T., Method to solve the nonlinear infinite horizon optimal control problem with application to the track control of a mobile robot
1703

3013

3519

3253

877

4245

2839

3741

4145

2127

4453

4177

1985

1367

255

3461

2033

4117

2169

1753

3619

2605

1329

2547

3387

2215

2049

1661

3725

2289

4177

1741

1713

561
Homer, M.E. \& Hogan, S.J., Impact dynamics of large dimensional systems

561

Hong, Y., see Chen

Hsieh, J.-G., see Lin

243

3151

Hsu, C.-H. \& Yang, T.-S., Periodic oscillations arising and death in delay-coupled neural loops

4015

Hsu, C.-H. \& Yang, T.-S., Stability of cellular neural networks with distributive time delays and noise perturbations

$\mathrm{Hu}$, H.Y., see Wang

$\mathrm{Hu}, \mathrm{W}$., see Liu

Huang, H. \& Cao, J., Master-slave synchronization of Lur'e systems based on time-varying delay feedback control

Huang, K., see Yang

Huang, L., see Duan

Huang, L., see Liu

Huang, X., Lam, J., Cao, J. \& Xu, S., Robust synchronization criteria for recurrent neural networks via linear feedback

Huang, Y., see Yang

Hui, J. \& Zhu, D.-M., Dynamics of SEIS epidemic models with varying population size

Hwang, D.-U., see Boccaletti

Hwang, D.U., see Brugioni

Ionescu, A., see Constantinescu

Iordache, M. \& Dumitriu, L., Time domain diakoptic analysis based on reduced-order state equations

Iordache, M., see Dumitriu

Ishiguro, K., see Lungarella

Ishikawa, Y., see Saito

Itoh, M. \& Chua, L.O., Advanced image processing cellular neural networks

Itoh, M. \& Chua, L.O., Boids control of chaos

Itoh, M. \& Chua, L.O., Oscillations on the edge of chaos via dissipation and diffusion

Iwanaga, H., see Sumida

Jacquir, S., Binczak, S. \& Bilbault, J.-M., Analytical determination of initial conditions leading to firing in nerve fibers

Jacquir, S., Binczak, S., Bilbault, J.M. \& Athias, P., A theoretical approach of the propagation through geometrical constraints in cardiac tissue

Jahed-Motlagh, M.R., Kia, B., Ditto, W.L. \& Sinha, S., Fault tolerance and detection in chaotic computers

Janowski, E.J., Kulenović, M.R.S. \& Nurkanović, Z., Stability of the kth order Lyness' equation with a period- $k$ coefficient

Jeng, J.-H., see Lin

Jiang, F., see Liu

Jiang, J., Han, M., Yu, P. \& Lynch, S., Limit cycles in two types of symmetric Liénard systems

Jorba, À., Tatjer, J.C., Núñez, C. \& Obaya, $\mathrm{R}$., Old and new results on strange nonchaotic attractors
535

2805

1735

4159

3929

3047

2705

2723

953

1513

2447

3431

3583

3625

3595

903

3373

1109

427

1531

283

3697

4417

1955

143

3151

1735

3895 
Juang, J., see Chang

Judd, K., see Nakamura

Judd, K., Galton's quincunx: Random walk or chaos?

Kabe, T., see Saito

Kahng, B., see Lee

Kalisky, T., see Braunstein

Kaltenbach, S., see Dolzmann

Kapranov, M., see Tomashevskiy

Karacs, K., see Chua

Karauz, B., see Kiliç

Kawakami, H., see Tsuji

Kawakami, H., see Tsuji

Kelber, K. \& Schwarz, W., Some design rules for chaos-based encryption systems

Keller, H.B., see Doedel

Kevrekidis, I.G., see Adomaitis

Kia, B., see Jahed-Motlagh

Kikombo, A.K., Oya, T., Asai, T. \& Amemiya, Y., Discrete dynamical systems consisting of singleelectron circuits

Kiliç, R. \& Karauz, B., Implementation of a laboratory tool for studying mixed-mode chaotic circuit

Kiliç, R. \& Yildirim, F., Experimental realizations of higher dimensional chaotic circuits including both floating and grounded inductors

Kiliç, R., SC-CNN based multifunction signal generator

Kilminster, D., see Nakamura

Kim, D., see Lee

Kim, H., see Ślot

Kitajima, H. \& Kurths, J., Forced synchronization in Morris-Lecar neurons

Klinker, T., see Holzhüter

Kohda, T., see Hane

Kohda, T., see Ono

Kohno, T., see Takemoto

Konishi, K., Amplitude death induced by a global dynamic coupling

Koppenberger, M., see Park

Kornatowski, 亡., see Ślot

Krachkovskyi, V., see Popovych

Krauskopf, B., see England

Krauskopf, B., see Rottschäfer

Krauskopf, B., see Sieber

Kravtsov, Yu.A., see Anosov

Krupa, M., see Golubitsky

Kulenović, M.R.S., see Janowski

Kuniyoshi, Y., see Lungarella

Kurths, J., see Allefeld

Kurths, J., see Kitajima

Kurths, J., see Pereira

Kurths, J., see Schelter

Kurths, J., see Tokuda

Kurths, J., see Wessel

Kurths, J., see Zou

Kuzmin, L., see Dmitriev

Kuznetsov, N.V., see Leonov

Kwong, S., see Sun

La Rosa, M., see Fortuna
1969

1741

4463

3373

2485

2215

1329

3669

3741

3633

837

985

3703

2625

1305

1955

3613

3633

607

4387

1741

2485

3171

3523

3607

3619

3645

459

2781

2281

3171

2517

805

1575

2579

1661

2595

143

903

3493

3523

3545

3551

3557

3325

4273

3443

1079

631

3035
Lai, D., A nonparametric statistical test for chaos: Cumulative periodogram under an order transformation

2815

Lakshmanan, M., see Palaniyandi

Lam, H.K. \& Leung, F.H.F., Stabilization of chaotic systems using linear sampled-data controller

Lam, H.K. \& Seneviratne, L.D., Synchronization of chaotic systems using neural-network-based controller

Lam, J., see Huang

Langford, W.F., see Harlim

Latora, V., see Boccaletti

Lawrance, A.J., see Baranovski

Lee, H.K., Goh, K.-I., Kahng, B. \& Kim, D., Internet data packet transport: From global topology to local queueing dynamics

Lehnertz, K., see Chernihovskyi

Lehnertz, K., see Osterhage

Lehnertz, K., see Osterhage

Lehnertz, K., see Staniek

Leonov, G.A. \& Kuznetsov, N.V., Time-varying linearization and the Perron effects

Letellier, C., Mendes, E.M.A.M. \& Mickens, R.E., Nonstandard discretization schemes applied to the conservative Hénon-Heiles system

Leung, F.H.F., see Lam

Li, C., see Guo

Li, C.-H. \& Yang, S.-Y., Exponential synchronization in drive-response systems of Hopfield-type neural networks with time delays

Li, C.-L., see Chang

Li, G., see Wen

Li, J. \& Chen, G., On a class of singular nonlinear traveling wave equations

Li, J.-B., see Chen

Li, Q., see Yang

Li, Q., see Yang

Li, Q., see Yang

Li, R., see Duan

Li, Y.C., Chaos in wave interactions

Lillo, F., see Tumminello

Lin, C., see Wen

Lin, S.-S., see Chan

Lin, Y.-L., Hsieh, J.-G. \& Jeng, J.-H., Robust template decomposition with restricted weights for cellular neural networks implementing an arbitrary Boolean function

Lin, Y.-T., see Chou

Lind, P.G., Mora, A., Haase, M. \& Gallas, J.A.C., Minimizing stochasticity in the NAO index

Lindberg, E., Tamaševičius, A., Mykolaitis, G. \& Bumelienè, S., Towards threshold frequency in chaotic Colpitts oscillator

Litak, G., Ali, M. \& Saha, L.M., Pulsating feedback control for stabilizing unstable periodic orbits in a nonlinear oscillator with a nonsymmetric potential

Liu, B. \& Yuan, Z., Exponential convergence of cellular neural networks with continuously distributed delays
4187

2021

2117

2723

2547

2447

1185

2485

3425

3305

3539

3729

1079

891

2021

1367

4167

1969

1753

4049

4395

583

3211

4205

3047

85

2319

1753

4099

3151

4337

3461

3449

2797

4403 
Liu, J. \& Min, L., Robust designs for grayscale global connectivity detection CNN templates

Liu, W., Tang, W.K.S. \& Chen, G., $2 \times 2$-Scroll attractors generated in a three-dimensional smooth autonomous system

Liu, X., Wang, J. \& Huang, L., Attractors of fourth-order Chua's circuit and chaos control

Liu, Z., Zhu, X., Hu, W. \& Jiang, F., Principles of chaotic signal radar

Llibre, J. \& Paşca, D., Periodic orbits near a heteroclinic loop formed by one-dimensional orbit and a two-dimensional manifold: Application to the charged collinear three-body problem

Llibre, J. \& Valls, C., Formal and analytic integrability of the Rossler system

Llibre, J., Ponce, E. \& Teruel, A.E., Horseshoes near homoclinic orbits for piecewise linear differential systems in $\mathbb{R}^{3}$

Llibre, J., see Corbera

Llibre, J., see Ferragut

Loiacono, D., see Dercole

Lombardo, D., see Arena

López, E., see Braunstein

Loskutov, A. \& Mironyuk, O., Time series analysis of ECG: A possibility of the initial diagnostics

Loskutov, A., see Dzhanoev

Lou, X. \& Cui, B., Global exponential stability conditions for delayed parabolic neural networks with variable coefficients

Lou, Y., Chen, F. \& Guan, J., Fingerprint feature extraction via CNN with Von Neumann neighborhood

Lu, Q., see Shi

Lü, J., see $Y u$

Lü, T. \& Zhang, X., Darboux polynomials and algebraic integrability of the Chen system

Lungarella, M., Ishiguro, K., Kuniyoshi, Y. \& Otsu, N., Methods for quantifying the causal structure of bivariate time series

Luo, X., Small, M., Danca, M.-F. \& Chen, G., On a dynamical system with multiple chaotic attractors

Lynch, S., see Jiang

Macabéa, J., Bifurcations in dynamics of Shepherd systems

Mahmoud, E.E., see Mahmoud

Mahmoud, G.M., Bountis, T. \& Mahmoud, E.E., Active control and global synchronization of the complex Chen and Lü systems

Maier-Paape, S., Mischaikow, K. \& Wanner, T., Structure of the attractor of the Cahn-Hilliard equation on a square

Malberg, H., see Wessel

Man, K.F., see Tang

Mansouri, N., see Boukabou

Mantegna, R.N., see Tumminello

Marchiori, M., Social search engines

Mariño, I.P., Zambrano, S., Sanjuán, M.A.F., Salvadori, F., Meucci, R. \& Arecchi, F.T., Adaptive procedure for the parameter estimation of a model of a $\mathrm{CO}_{2}$ chaotic laser

3639
2827

Markus, M., see Grete

Marquié, P., see Morfu

Marquié, P., see Morfu

4153

2705

1735

2175

3289

1171

3295

2159

2435

107

2215

3709

3603

4409

4145

4355

1785

903

3235

2169

545

4295

4295

1221

3325

923

2141

2319

2355 analysis

Min, L., see Liu

Monti, M., see Castro

Mora, A., see Lind network media feedback

Motoike, I.N., see Oya
Martínez, J.A., see Chacón

1007

3535

3721

Masdemont, J.J., see Gidea

625

1151

Matsuoka, Y., see Saito

Mendes, E.M.A.M., see Campos

3373

3199

891

Meškauskas, M., Tamaševičius, A. \& Pyragas, K., Analogue signal predictor: Frequency-domain

3715

3657

3455

Meškauskas, M., see Tamaševičius

3431

3639

Meucci, R., see Mariño

Miccichè, S., see Tumminello

2319

Mickens, R.E., see Letellier

891

Milanesi, L., see Remondini

2477

Miliotis, A., see Simonotto

3229

Miller, D.A., see Grassi

Mironyuk, O., see Loskutov

Mischaikow, K., see Maier-Paape

Miyazaki, S. \& Nagashima, Y., Network as a chaotic dynamical system

Moiola, J.L., see Robbio

Montalbán, L.S., Henttu, P. \& Piché, R., Recurrence quantification analysis of electrochemical noise data during pit development

Moreno, Y., see J. Gómez-Gardeñes

Morfu, S., Bossu, J. \& Marquié, P., Experiments on an electrical nonlinear oscillators

1337

2827

3709

1221

3529

1623

Morfu, S. \& Marquié, P., Effect of noise and structural inhomogeneities in reaction-diffusion

Morgül, Ö., On the stabilization of periodic orbits for discrete time chaotic systems by using scalar

Mormann, F., see Osterhage

Morrison, P.J., see Portela

Moukam Kakmeni, F.M. \& Bowong, S., Chaos and robust adaptive synchronization in a nonlinear emitter-receiver system

Mozelli, L.A., see Campos

Müller, M., see Allefeld

Muñoz-Almaraz, F.J., Freire, E. \& Galán-Vioque, J., Bifurcation behavior of the Furuta pendulum

965

3461

2501

3535

Muscato, G., see Buscarino

Muthuswamy, B., see Chang

Mykolaitis, G., see Lindberg

Nagashima, Y., see Miyazaki

Nakamura, T., Hirata, Y., Judd, K., Kilminster, D. \& Small, M., Improved parameter estimation from noisy time series for nonlinear dynamical systems 
Nana Nbendjo, B.R., see Yamapi

Nandi, A., see Prasad

Neerhoff, F.L., see van der Kloet

Neretti, N., see Remondini

Nicolosi, D., see Fortuna

Nishio, Y., see Uwate

Nistri, P., see Di Marco

Nitescu, M., see Constantinescu

Núñez, C., see Jorba

Nurkanović, Z., see Janowski

Nutter, N.G., see Davis

Obaya, R., see Jorba

Ono, A. \& Kohda, T., Solvable three-dimensional rational chaotic map defined by Jacobian elliptic functions

Osinga, H.M., see England

Osterhage, H. \& Lehnertz, K., Nonlinear time series analysis in epilepsy

Osterhage, H., Mormann, F., Staniek, M. \& Lehnertz, K., Measuring synchronization in the epileptic brain: A comparison of different approaches

Otsu, N., see Lungarella

Oya, T., Motoike, I.N. \& Asai, T., Single-electron circuits performing dendritic pattern formation with nature-inspired cellular automata

Oya, T., see Kikombo

Paffenroth, R.C., see Doedel

Palacios, A., Blomgren, P. \& Gasner, S., Bifurcation analysis of hopping behavior in cellular pattern-forming systems

Palacios, A., see Gasner

Palaniyandi, P. \& Lakshmanan, M., Controlled parameter modulations in secure digital signal transmissions

Palhares, R.M., see Campos

Pan, Y. \& Billings, S.A., Model validation of spatiotemporal systems using correlation function tests

Pancioni, L., see Di Marco

Pantano, P., see Bilotta

Pantano, P., see Bilotta

Pantano, P., see Bilotta

Pantano, P., see Bilotta

Pantano, P., see Bilotta

Pantano, P., see Bilotta

Paolini, E.E., see Robbio

Pardo, W.B., see Castro

Park, J., Celma, O., Koppenberger, M., Cano, P. \& Buldú, J.M., The social network of contemporary popular musicians

Parlitz, U., see Tokuda

Paşca, D., see Llibre

Patanè, L., see Arena

Peng, M. \& Yuan, Y., Synchronization and desynchronization in a delayed discrete neural network

Perc, M., Uncertainties facilitate aggressive behavior in a spatial hawk-dove game
1343

3397

3675

2477

3035

3565

3127

3583

3895

143

3513

3895

3645

805

3305

3539

903

3651

3613

2625

509

2765

4187

3199

4323

3127

1

293

657

1017

1383

1801

1623

965

2281

3557

2175

107

791

4223
Pereira, T., Baptista, M.S., Kurths, J. \& Reyes, M.B., Onset of phase synchronization in neurons with chemical synapse

Petermann, T., see De Los Rios

Pich, C., see Brandes

Piché, R., see Montalbán

Ponce, E., see Freire

Ponce, E., see Llibre

Popovych, O.V., Krachkovskyi, V. \& Tass, P.A., Twofold impact of delayed feedback on coupled oscillators

Portela, J.S.E., Caldas, I.L., Viana, R.L. \& Morrison, P.J., Diffusive transport through a nontwist barrier in tokamaks

Portela, J.S.E., Caldas, I.L., Viana, R.L. \& Sanjuán, M.A.F., Fractal and Wada exit basin boundaries in tokamaks

Prasad, A., Nandi, A. \& Ramaswamy, R., Aperiodic nonchaotic attractors, strange and otherwise

Pyragas, K., see Meškauskas

Pyragas, K., see Tamaševičius

Pyragienè, T., see Tamaševičius

Pyragienè, T., see Tamaševičius

Qingduan, F., see Zengrong

Qiu, S.-S., see Chen

Rademacher, J.D.M. \& Scheel, A., Instabilities of wave trains and turing patterns in large domains

Radwan, A., Soliman, A.M. \& Elwakil, A.S., 1-D digitally-controlled multiscroll chaos generator

Ramaswamy, R., see Prasad

Ramírez Ávila, G.M. Guisset, J.-L. \& Deneubourg, J.-L., Influence of uniform noise on two light-controlled oscillators

Rasmusson, R.L., see Bondarenko

Remondini, D., Neretti, N., Franceschi, C., Tieri, P., Sedivy, J.M., Milanesi, L. \& Castellani, G.C., Networks from gene expression time series: Characterization of correlation patterns

Reyes, E.G. \& Sanchez, G., Explicit solutions to the Kaup-Kupershmidt equation via nonlocal symmetries

Reyes, M.B., see Pereira

Richter, P.H., see Dullin

Rinaldi, S., see Dercole

Ringwood, J., see Fay

Rizzo, A., see Buscarino

Robbio, F.I., Paolini, E.E., Moiola, J.L. \& Chen, G., Harmonic distortion analysis based on Hopf bifurcation theorem and fast Fourier transform

Rodgers, G.J., see Tadić

Rodríguez-Luis, A.J., see Algaba

Romance, M., see Criado

Romano, M.C., see Zou

Romanov, V.A., see Doedel

3545

2331

2303

3725

445

1171

2517

1589

4067

3397

3715

3657

3455

3657

2109

1609

2679

227

3397

4453

381

2477

2749

3545

3013

2435

3691

2411

Ros, J., see Freire

Rosa, Jr., E., see Castro

Rosa, Jr., E., see Davis
1623

2363

1997

2289

4273

2625

445

965

3513 
Rosa, R., see Giannerini

Rosas-Casals, M., Valverde, S. \& Solé, R.V., Topological vulnerability of the European power grid under errors and attacks

Rosen, M., see Cabeza

Rosvall, M. \& Sneppen, K., Networks and our limited information horizon

Rottschäfer, V. \& Krauskopf, B., The ECMbackbone of the Lang-Kobayashi equations: A geometric picture

Routh, A.F., see Zhao

Routh, A.F., see Zhao

Roy, P.K., see Dana

Saha, L.M., see Litak

Saito, T., Kabe, T., Ishikawa, Y., Matsuoka, Y. \& Torikai, H., Piecewise constant switched dynamical systems in power electronics

Saito, T., see Akatsu

Salvadori, F., see Mariño

Sanchez, G., see Reyes

Sandstede, B., Evans functions and nonlinear stability of traveling waves in neuronal network models

Sanjuán, M.A.F., see Buldú

Sanjuán, M.A.F., see Donner

Sanjuán, M.A.F., see Mariño

Sanjuán, M.A.F., see Portela

Sardanyés, J. \& Solé, R.V., Chaotic stability in spatially-resolved host-parasite replicators: The red queen on a lattice

Saso, Y., see Gotoda

Sbitnev, V.I., see Chua

Sbitnev, V.I., see Chua

Scheel, A., see Rademacher

Schelter, B., Winterhalder, M., Timmer, J. \& Kurths, J., Phase synchronization and coherence analysis: Sensitivity and specificity

Schelter, B., see Winterhalder

Schmidt, S., see Dullin

Schnegg, M. \& Stauffer, D., Dynamics of networks and opinions

Schwarz, W., see Kelber

Sedivy, J.M., see Remondini

Seehafer, N., see Donner

Seneviratne, L.D., see Lam

Shang, D., see Han

Shen, S., see Guan

Shi, X., Lu, Q. \& Chen, G., Anti-phase synchronization of inhibitorily coupled neurons

Shin, J., see Chua

Shin, J., see Chua

Sicurella, G., see Fortuna

Sieber, J. \& Krauskopf, B., Control-based continuation of periodic orbits with a time-delayed difference scheme

Simonotto, J.D., Furman, M.D., Ditto, W.L., Miliotis, A., Spano, M.L. \& Beaver, T.M., Dynamic transmurality: Cardiac optical mapping reveals waves travel across transmural ablation lines

Sinha, S., see Jahed-Motlagh
169

2465

1599

2509

1575

153

1687

3437

2797

3373

3571

3639

2749

2693

3507

3589

3639

4067

589

2185

2839

3741

2679

3551

3735

3013

2399

3703

2477

3589

2117

4117

4245

4355

2839

3741

3035

2579

3229

1955
Śliwa, I., Szlachetka, P. \& Grygiel, K., Chaotic beats in a nonautonomous system governing second-harmonic generation of light

3253

Ślot, K., Kornatowski, Ł., Dȩbiec, P. \& Kim, H., Generation of complex stochastic textures using cellular neural networks

3171

3235

1741

2509

2465

589

227

1637

Song, C.Z., see Zhang

Song, Y. \& Banks, S.P., Dynamical systems on three manifolds. Part II: Three-manifolds, Heegaard splittings and three-dimensional systems

Song, Y., Banks, S.P. \& Diaz, D., Dynamical systems on three manifolds. Part I: Knots, links and chaos

Sorrentino, F., di Bernardo, M. \& Garofalo, F., Synchronizability and synchronization dynamics of weighed and unweighed scale free networks with degree mixing

Sorrentino, F., see di Bernardo

Spano, M.L., see Simonotto

Sparro, M., see Colacchio

Sporns, O., see Costa

Sprott, J.C. \& Chlouverakis, K.E., Labyrinth chaos

2085

2073

Sreenivasan, S., see Braunstein

Staniek, M. \& Lehnertz, K., Parameter selection for permutation entropy measurements

Staniek, M., see Osterhage

Stanley, H.E., see Braunstein

Starkov, K.E., Estimation of the domain containing all compact invariant sets of the optically injected laser system

Stauffer, D., see Schnegg

Stewart, I., see Antoneli

Stewart, I., Elimination of multiple arrows and self-connections in coupled cell networks

Stoop, R. \& Wagner, C., Neocortex's architecture optimizes computation, information transfer and synchronizability, at given total connection length

Stoop, R., see Wagner

Stranges, F., see Bilotta

Stranges, F., see Bilotta

Stranges, F., see Bilotta

Stranges, F., see Bilotta

Stranges, F., see Bilotta

Stranges, F., see Bilotta

Sumida, T., Iwanaga, H. \& Tahara, T., Numerical indication of structural stability in dynamical systems and its application to clinical study

Sun, C., see Han

Sun, J., see Wan

Sun, J., see Zhou

Sun, J., see Zhou
2033

2419

3499

3229

1911

2387

2097

2215

3729

3539

2215

4213

2399

935

99

3409

1

293

657

1017

1383

1801

3219

129

3099 
Sun, S. \& Kwong, S., Stochastic resonance signal processor: Principle, capacity analysis and method

Sun, Y. \& Yin, Y., The connectedness locus of a family of real biquadratic polynomials

Suykens, J.A.K., see Hillier

Szlachetka, P., see Śliwa

Tadé, M.O., see Zhang

Tadić, B., Rodgers, G.J. \& Thurner, S., Transport on complex networks: Flow, jamming and optimization

Tahara, T., see Sumida

Takahashi, M., Asai, T., Hirose, T. \& Amemiya, Y., A CMOS reaction-diffusion device using minority-carrier diffusion in semiconductors

Takemoto, T., Kohno, T. \& Aihara, K., Circuit implementation and dynamics of a twodimensional MOSFET neuron model

Tamaševičius, A., Pyragienè, T., Pyragas, K., Bumelienè, S. \& Meškauskas, M., Numerical treatment of educational chaos oscillator

Tamaševičius, A., see Lindberg

Tamaševičius, A., see Meškauskas

Tamaševičius, A., Pyragienè, T. \& Meškauskas, M., Two-scroll attractor in a delay dynamical system

Tang, C., see Guan

Tang, K.W., Tang, W.K.S. \& Man, K.F., A chaos-based pseudo-random number generator and its application in voice communications

Tang, W.K.S., see Liu

Tang, W.K.S., see Tang

Tang, W.K.S., see $Y u$

Tass, P.A., see Popovych

Tatjer, J.C., see Jorba

Tebaldi, C., see Colacchio

Teixeira, M.A., see Ferragut

Teng, Z., see Gao

Teo, K.-L., see Xie

Teruel, A.E., see Llibre

Thiel, M., see Zou

Thurner, S., see Tadić

Tian, L. \& Xu, G., Sudden occurrence of chaos in nonsmooth maps

Tian, Y.-C., see Zhang

Tieri, P., see Remondini

Tigan, G. \& Astolfi, A., A note on a piecewiselinear Duffing-type system

Timmer, J., see Schelter

Timmer, J., see Winterhalder

Tokuda, I., Kurths, J. \& Parlitz, U., Ensemble approach for recovering phase synchronization from time series

Tomashevskaya, M., Spline approximation application to reconstruction of discrete map functions

Tomashevskiy, A. \& Kapranov, M., Fractal distribution of reverse iterations in maps with chaotic dynamics

Torikai, H., see Akatsu
631

4219

4177

3253

3281

2363

283

1713

459

3657

3449

3715

3455

4245

923

4153

923

3951

2517

3895

1911

2159

521

3183

1171

4273

2363

271

3281

2477

4425

3551

3735

3557

3663
Torikai, H., see Saito

3373

Torres, L.A.B., see Campos

3199

1609

Tsonis, A.A., Reconstructing dynamics from observables: The issue of the delay parameter revisited

Tsuji, S., Ueta, T. \& Kawakami, H., Bifurcation analysis of current coupled BVP oscillators

Tsuji, S., Ueta, T., Kawakami, H., Fujii, H. \& Aihara, K., Bifurcations in two-dimensional Hindmarsh-Rose type model

Tumminello, M., Coronnello, C., Lillo, F., Miccichè, S. \& Mantegna, R.N., Spanning trees and bootstrap reliability estimation in correlationbased networks

Ueta, T., see Tsuji

Ueta, T., see Tsuji

Umana, E., see Fortuna

Urías, J., see Campos-Cantón

Uwate, Y. \& Nishio, Y., Synchronization phenomena in Van Der Pol oscillators coupled by a time-varying resistor

Valls, C., see Llibre

Valverde, S., see Rosas-Casals

van der Kloet, P., Neerhoff, F.L. \& Waning, N.H., Some analytic calculations of the characteristic exponents

Vanderbauwhede, A., see Doedel

Vandewalle, J., see Hillier

Vecchio, P., see Grassi

Vecchio, P., see Grassi

Verduzco, F., Control of codimension one stationary bifurcations

Verhulst, F., Periodic solutions and slow manifolds

Vespignani, A., see Colizza

Viana, R.L., see Portela

Viana, R.L., see Portela

von Haeseler, F., see Barbé

Wagemakers, A., see Buldú

Wagner, C. \& Stoop, R., Neocortex's small world of fractal coupling

Wagner, C., see Stoop

Walkenstein, J.A., see Castro

Wan, L., Zhou, Q. \& Sun, J., Mean value exponential stability of stochastic reaction-diffusion generalized Cohen-Grossberg neural networks with time-varying delay

Wan, L., see Zhou

Wang, B., Dynamical behavior of the almostperiodic discrete Fitzhugh-Nagumo systems

Wang, J., see Duan

Wang, J., see Liu

Wang, Q.-G., see Wen

Wang, Z., Fan, H. \& Aihara, K., An associative network with chaotic neurons and dynamic synapses

Wang, Z.H. \& Hu, H.Y., Pseudo-oscillator analysis of scalar nonlinear time-delay systems near a Hopf bifurcation
4229

837

985

2319

837

985

3035

199

3565

3289

2465

3675

2625

4177

1323

1703

575

2533

2491

1589

4067

1265

3507

3409

2257

965

3219

3099

1673

3047

2705

1753

3085

2805 
Waning, N.H., see van der Kloet

Wanner, T., see Maier-Paape

Webber, Jr., C.L. \& Zbilut, J.P., Recurrence quantifications: Feature extractions from recurrence plots

Webber, Jr., C.L., see Zbilut

Wei, H.-C., see Chou

Wei, J. \& Fan, D., Hopf bifurcation analysis in a Mackey-Glass system

Wen, G., Wang, Q.-G., Lin, C., Li, G. \& Han, X., Chaos synchronization via multivariable PID control

Wessel, N., Malberg, H., Bauernschmitt, R. \& Kurths, J., Nonlinear methods of cardiovascular physics and their clinical applicability

Winterhalder, M., Schelter, B. \& Timmer, J., Detecting coupling directions in multivariate oscillatory systems

Winterhalder, M., see Schelter

Witt, A., see Donner

Wong, S.-C., see Chen

$\mathrm{Wu}, \mathrm{Z}$., see Braunstein

Xiao, M. \& Cao, J., Bifurcation analysis and chaos control for Lü system with delayed feedback

Xie, D., see Gao

Xie, L., Teo, K.-L. \& Zhao, Y., Invariant manifold with complete foliations and chaotification analysis for a kind of PDEs with boundary coupling

$\mathrm{Xu}, \mathrm{G} .$, see Tian

$\mathrm{Xu}$, J.-X., see Zhang

$\mathrm{Xu}, \mathrm{S}$., see Huang

Yalçın, M.E., Increasing the entropy of a random number generator using n-scroll chaotic attractors

Yamaguchi, T., see Yamamoto

Yamamoto, T., Amemiya, T. \& Yamaguchi, T., Learning and recalling of phase patterns in coupled BVP oscillators with time delay

Yamapi, R. \& Bowong, S., Dynamical states in a ring of four mutually coupled selfsustained electrical systems with time periodic coupling

Yamapi, R., Nana Nbendjo, B.R. \& Enjieu Kadji, H.G., Dynamics and active control of motion of a driven multi-limit-cycle Van der Pol oscillator

Yan, Z. \& Yu, P., Globally exponential hyperchaos (lag) synchronization in a family of modified hyperchaotic Rössler systems

Yang, F., Li, Q. \& Zhou, P., Horseshoe in the hyperchaotic MCK circuit

Yang, Q., Chen, G. \& Huang, K., Chaotic attractors of the conjugate Lorenz-type system

Yang, S.-Y., see $L i$

Yang, T.-S., see Hsu

Yang, T.-S., see Hsu

Yang, X.-S. \& Huang, Y., Chaos and two-tori in a new family of 4-CNNs
Yang, X.-S. \& Li, Q., A horseshoe in a cellular neural network of four-dimensional autonomous ordinary differential equations

Yang, X.-S. \& Li, Q., Chaos in simple cellular neural networks with connection matrices satisfying Dale's rule

Yang, X.-S., see Yuan

Yao, M.H. \& Zhang, W., Shilnikov-type multipulse orbits and chaotic dynamics of a parametrically and externally excited rectangular thin plate

851

Yao, W., see $Y u$

Yildirim, F., see Kiliç

Yin, Y., see Sun

2791

607

Y., Yao, W. \& Chen, G., Analysis on topological properties of the Lorenz and the Chen attractors using $G C M$

2791

4117

Yu, P., see Han

$\mathrm{Yu}, \mathrm{P}$. , see Jiang

$\mathrm{Yu}, \mathrm{P}$. , see Yan

$\mathrm{Yu}, \mathrm{P} .$, Local and global bifurcations to limit cycles in a class of Liénard equation

Yu, S., Lü, J. \& Chen, G., A module-based and unified approach to chaotic circuit design and its applications

583

4381

2169

1759

183

1785

Yu, S., Tang, W.K.S. \& Chen, G., Generation of $n \times m$-scroll attractors under a Chua-circuit framework

$\mathrm{Yu}$, W. \& Cao, J., Stability and Hopf bifurcation on a two-neuron system with time delay in the frequency domain

Yuan, Q. \& Yang, X.-S., Computer assisted verification of chaos in three-neuron cellular neural networks

Yuan, Y., see Peng

Yuan, Z., see Liu

Zambrano, S., see Mariño

Zbilut, J.P. \& Webber, Jr., C.L., Recurrence quantification analysis: Introduction and historical context

Zbilut, J.P., see Webber, Jr.

Zeng, L. \& Zhao, Y., Characterization of static bifurcations for maps in the frequency domain

3951

4381

791

4403

3639

3477

3467

975

Zengrong, L., Chengdong, D. \& Qingduan, F., Multicenter network and synchronization

2109

Zgliczyński, P., see Galias

Zhang, F., see Heidel

4261

2049

Zhang, G., Chen, G., Chen, T. \& D'Amico, M.B., Dynamical analysis of a networked control system

61

Zhang, G.-J. \& Xu, J.-X., The correspondence between stochastic resonance and bifurcation of moment equations of noisy nonlinear dynamical system

Zhang, T. \& Zhu, D., Bifurcations of homoclinic orbit connecting two nonleading eigendirections

Zhang, T., Tian, Y.-C. \& Tadé, M.O., On the number of zeros of the Abelian integrals for a class of perturbed Liénard systems 
Zhang, W. \& Song, C.Z., Higher-dimensional periodic and chaotic oscillations for viscoelastic moving belt with multiple internal resonances

Zhang, W., see Yao

Zhang, X., see Lü

Zhang, Z. \& Chen, G., Chaotic liquid shaker: Design, implementation and application

Zhao, Y., Billings, S.A. \& Routh, A.F., Identification of excitable media using cellular automata models

Zhao, Y., Billings, S.A. \& Routh, A.F., Identification of the Belousov-Zhabotinskii reaction using cellular automata models

Zhao, Y., see Xie

Zhao, Y., see Zeng

Zheng, W., see Han

Zhong, Q., see Chen

Zhou, P., see Yang

4205

1637

851

2739

4443

153

3183

975

4117

243
Zhou, Q., Sun, J. \& Chen, G., Global exponential stability and periodic oscillations of reaction-diffusion BAM neural networks with periodic coefficients and general delays

Zhou, Q., Wan, L. \& Sun, J., Exponential stability of reaction-diffusion fuzzy recurrent neural networks with time-varying delays

3099

3219

823

1513

1735

999

Zhu, Z., see Chen

Zou, Y., Thiel, M., Romano, M.C. \& Kurths, J., Analytical description of recurrence plots of dynamical systems with nontrivial recurrences 